\title{
FIRST STEPS IN INTEGRATING MICRO- ASSEMBLY FEATURES INTO INDUSTRIALLY USED DFA SOFTWARE
}

\author{
Timo Salmi* and Juhani Lempiäinen** \\ *VTT Industrial Systems, P.O.Box 1702, FIN - 02044 VTT, Finland * Deltatron Oy, Soidintie \\ 14, FIN - 00700 Helsinki, Finland
}

Abstract: This paper discusses the problems of micro-scale part manipulation and assembly. Parts less than $10 \times 10 \times 10 \mathrm{~cm} 3$ in size need special attention when they are designed. There is a need for handling tools, feeding systems, and fastening methods that resemble macro-scale industrial systems but are of a special nature. No effort has been made prior to this presentation to collect the data in any systematic form for designers. The Finnish $D F A-T o o l$ software version 3.0 is the first attempt to try to formulate this problematic in a systematic and suitable presentation for product designers.

Key words: Micro-Assembly, Design for Assembly, DFA, Assemblability analysis

\section{THE DESIGN FOR ASSEMBLY APPROACH}

Design for Assembly (DFA) rules have over the years been recognized as a powerful and valuable methodology for developing and analyzing producible products. The DFA issue has been approached in different ways. First, it has been given recommendations and rules on how to design products or product components so that they are easy to assemble manually or automatically (e.g. Andreasen et al 1983). Easy automatic assembly means that the product primarily is possible to assemble automatically. The assembly process can be performed by quite simple and commonly accepted known technology, which means that the process is expected to be very reliable and well known. The second approach is methods for analysing the 
assemblability of products (e.g. Boothroyd et al 1983). Typically the results indicate producibility in the form of an index and an estimated assembly time. Nowadays, most analysis methods have been developed as software tools. The third approach is to study how to develop the design process so that producibility issues are optimally considered. All of these aspects should be implemented if an industrial company is to benefit from DFA. The significance of DFA may be summarized as a precondition for automatic assembly and/or profitable high volume production. With the use of DFA methods during the early design phase, there is a good chance of avoiding the assembly problems that usually emerge during production start-up and even later if DFA methodology is not used.

The DFA design rules and DFA tools are based on the traditional assembly and on the technical solutions that are commonly used in that area. There are no commercialized DFA tools that can give advice in the microspecific assembly problems.

This paper considers the problems of creating DFA rules for micro-part assembly and introduces the development of a method to handle some problems that are typical especially of micro-assembly. The methods have also been developed in the form of software and included in an industrially used DFA tool for industrial field tests.

\section{DIFFERENCES BETWEEN MICRO AND MACRO ASSEMBLY}

When entering the micro world there are two main issues that have special importance: The physical behaviour of parts is changing, as are the feeding, manipulation, and insertion techniques used. When scaling down, the gravitational and inertial forces may become insignificant compared to adhesive forces such as electrostatic, surface tension or van der Waal's forces. The micro-forces that exceed gravity can, for example, have the effect that as a gripper approaches a part, electrostatic attraction causes the part to jump off the surface into the gripper with an orientation only dependent on initial charge distributions. And when the part is placed in the assembly position, it may remain in the gripper tool instead of connecting to the base part. These phenomena make accurate placement of the micro-part difficult.

Micro-assembly in many cases meets the technical limitations of traditional technology. Partly because of the limitations and partly because of the new phenomenon, the following new production technologies have been introduced for micro-assembly: 
- New grippers, such as (Tichem et al 2002, Lambert et al 2004) contactless grippers, capillary grippers

- New feeding methods, such as different types of reel magazines, adhesive and blister tape cavities, magazines, standard pallets and gel packs, blade feeders, hopper feeders with indexing capabilities

- The use of machine vision technology in different phases of the assembly process. Machine vision is used very often in micro-assembly to compensate various types of positioning errors.

- New joining methods.

\section{DESIGN FOR MICRO-ASSEMBLY}

The majority of traditional DFA rules are valid in micro-assembly. This question was studied more deeply by Eskilander \& Salmi in 2004. However, some new guidelines are needed especially for micro-assembly. Most design rules have been formalized from the analyses of stepwise process chains and commonly used assembly equipment, and from the study of function principles and technical limitations. There are at least two kinds of difficulties when creating new design rules for micro-assembly. First, the technical limitations, e.g. size of the part, are not easy to determine. Although there are some common practices, someone will often cross the line with a slightly different technical solution. Therefore a huge number of tests or published research results would be needed. Second is the divergence of technical solutions. The new phenomenon has raised new technical solutions alongside the traditional ones. Many of the innovations are either in the laboratory phase or not widely used. And micro-assembly technologies are developing rapidly. The different technologies also place different requirements on product design. When there are several different potential strategies for solving an assembly problem, it is not easy to formulate design rules. In this kind of situation it should be considered for which purposes these rules should be formulated. It would seem that integration of the product design, the process and the production equipment is even more essential in the micro world than in traditional assembly. Thus the product should be designed to be manufactured with a particular method and manipulated with a particular handling method, and so on. The requirement that the product should be assembled or manufactured easily with whatever commonly used method is too tight. The interaction between product and production design is such that product features limit possible handling technologies, and on the other hand the selected handling technology places requirements on the product design. 
In practical situations, the handling of parts is one of the key issues when designing miniaturized parts. The rules of micro-parts assembly actually resemble those for automated assembly, because manipulating parts of any size smaller than $10 \times 10 \times 10 \mathrm{~mm}^{3}$ must be done with the help of assembly grasping tools.

When scaling down the products and parts, it is very difficult for product designers and assembly system designers to realize when the limit is reached at which the gravitation force is no longer the most prominent force in the manipulation of a part. The need for clean, aseptic, and antistatic product assembly work conditions is often highlighted in small part assembly. That is reflected in the need for assembly gloves to cover hands, extra work operations due to the spraying of ionised air, and the special materials to ensure ground potential around the workplace. The need to ensure free sight at the point of assembly also has another meaning, as human sight resolution is not enough to guarantee the success of assembly operation.

The ultimate need for aseptic handling of parts raises new challenges even when grasping tools are not utilized. The use of rubber or cotton gloves in any case reduces the natural manual touch in assembly work. The practical speed reduction has been found to be around $10 \%$ of the assembly manipulation speed.

Some rules of thumb for micro-part manipulation can easily be generated for the appearance of the part (additional to the numerical entries of size and weight):

- Does it have a stable and indexed position on feeding?

- Does it have a relatively flat surface to grasp with vacuum/Bernoulli?

- Does the flat surface cover a major area of the part to grasp with vacuum/Bernoulli?

- Does the stiffness of the part allow grasping from the sides?

- Are the part sides parallel to each other so that finger-force grasping is possible?

- Does the part surface or shape allow any grasping force?

When interviewing companies on the joining methods used in their micro-assemblies, screwing is the most prominent assembly fastening method used for parts. This is no surprise, since $80 \%$ of the fastenings on the macro-scale are also done with screws. On the micro-scale there is thus a huge need to estimate the scaling factor and time constraints. When scaling screwing operations down, it seems obvious that all the design rules apply as they are of macro-scale down to a screw size of $0.8 \mathrm{~mm}$ in diameter. That seems to be the ultimate limit for feeding at the moment. Around this limit the torque control is difficult to obtain (around $1 \mathrm{Ncm}$ ) and the mechanical 
stiffness of the parts also limit the speed of screwing (from $1200 \mathrm{rpm}$ down to $300 \mathrm{rpm}$ ), thus causing major slowing down of the assembly process.

\section{MICRO-ASSEMBLY FEATURES IN A DFA TOOL}

The engineering office Deltatron Ltd has developed a software product for assemblability analysis called $\boldsymbol{D F A - T o o l} \otimes$. The most modern version of the software, v3.0, now includes some of the constraints and rules of micropart assembly described above. It also gives a hint when some other force than gravitation is most prominent in the assembly operation. This helps the designers to understand the character of the assembly operation and paves the way to selection of the most feasible assembly tool. This information is essential both in manual and in robotized assembly.

By asking the part appearance questions, it is possible to evaluate what is the most prominent force in the assembly and which grasping methods are still available for this particular part. The grasping forces for micro-parts are well presented by Fukuda \& Arai [1999] and Böhringer et al [1999]. They are roughly modelled in the DFA-Tool software. Based on the same database, the answers to the above questions on part appearance and nature will generate a list of possible grasping methods.

When selecting the screw type and size in the assembly construction, the software calculates the most feasible assembly time of the screw. In multiple screwing operations with automated feeding, DFA-Tool manages the extra manipulation times.

The software tool for micro-assemblability is one way of collecting know-how of common practices that the industry currently utilizes for micro-part manipulation. The development of this tool is an iterative process, and the interaction between the industrial user community of this technology and the software developers is the key element. In this case the beta-testers of this study are the world leading electronics and optoelectronics manufacturing companies. The DFA-Tool beta-tested version 3.0 will be introduced at the IPAS seminar in 2006. 


\section{REFERENCES}

1. Andreasen, M.,M., Kähler, S., Lund, T., Design For Assembly, Design for Assembly. Berlin, Heidelberg, New York, Tokio; IFS Publications Ltd and Springer-Verlag. $189 \mathrm{~s}$. ISBN 0-903608-35-9, 1983.

2. Boothroyd, G., Dewhurst, P., Design For Assembly, Handbook. Amherst: University of Massachusetts. 1983.

3. Böhringer, Karl F., Fearing Ronald S., Goldberg, Ken Y. Microassembly. In Handbook of Industrial Robotics, Second Edition, edited by Nof, Shimon Y. John Wiley \& Sons, Inc. NEw York, Chicester, Weinheim, Brisbane, Singapore, Toronto.. 1999. pp. 1045 - 1066.

4. Eskilander, Stephan; Salmi, Timo, Are traditional DFA methods valid in micro assembly? Proceedings of the International Precision Assembly Seminar (IPAS2004), Bad Hofgastein, 11-13 Febr, 2004. The Precision Manufacturing Group, School of Mechanic, Materials, Manufacturing Engineering and Management, The University of Nottingham (2004), pp. 19 - 26.

5. Fukuda, Toshio, Arai, Fumihito, Microrobotics, In Handbook of Industrial Robotics,, Second Edition, edited by Nof, Shimon Y. John Wiley \& Sons, Inc. NEw York, Chicester, Weinheim, Brisbane, Singapore, Toronto.. 1999. pp. $187-198$.

6. Lambert, P., Vandaele, V., Delchambre, A., Non-Contact Handling in Micro-assembly: State of the Art. Proceedings of the International Precision Assembly Seminar (IPAS2004), Bad Hofgastein, Austria, 11-13 Febr. 2004. The Precision Manufacturing Group, School of Mechanic, Materials, Manufacturing Engineering and Management, The University of Nottingham (2004) pp. 67 - 76.

7. Tichem, Marcel, Lang, Defeng, Karpuschewski, A Classification Scheme for Quantative Analysis of Micro-Grip Principles; Proceedings of the International Precision Assembly Seminar IPAS'2003, Bad Hofgastein, Austria, 17-19 March 2003, pp. 71 - 78. 